\title{
El lenguaje de las tecnologías en la formación de maestros
}

\section{The language of technologies in teacher education}

\author{
Nuria Andreu Ato \\ e-mail: nuria.andreu@uchceu.es \\ Universidad CEU Cardenal Herrera. España
}

Rocío López-García-Torres

e-mail: rocio.lopez@uchceu.es Universidad CEU Cardenal Herrera. España

Elia Saneleuterio id

e-mail: elia.saneleuterio@uv.es

Universitat de València. España

\section{Resumen}

Este trabajo pretende reflexionar sobre las implicaciones educativas que hoy día tiene la competencia mediática en los procesos de enseñanza-aprendizaje. La evolución de las TIC y, sobre todo, de la tecnología móvil, ha provocado una revolución comunicativa educativa y social que afecta al mundo educativo; los contextos educativos son cambiantes y evolucionan rápido, teniendo que adaptarse a los cambios socioculturales, tecnológicos, etc. Se contemplan tres resortes, a modo de estudio de revisión: los estudios sobre la competencia mediática desde el ámbito neurológico, desde la antropología conectivista y desde la perspectiva del proceso de enseñanzaaprendizaje. Se aportan tres conclusiones principales: los cambios en los modos de comunicación humana han fomentado el uso cotidiano de un lenguaje digital que no puede ser obviado en las aulas y que se concreta en la necesidad de desarrollar la competencia mediática; siendo imprescindible para lograrlo la incorporación de las TIC en las aulas, que repercute asimismo en la mejora de funciones ejecutivas básicas que potencian los aprendizajes; finalmente, la formación docente presenta una tendencia creciente hacia el compromiso para responder adecuadamente a las exigencias de la educación de un mundo tecnologizado, también contemplado en las leyes educativas actuales y cuya complementariedad puede interpretarse como garantía hacia un desarrollo adecuado de la competencia mediática en las generaciones del futuro que se formen en un uso fluido y responsable de las TIC y las TAC.

Palabras clave: alfabetización digital; competencia mediática; comunicación digital; sociedad tecnológica; tecnología educativa.

\section{Abstract}

This work aims to reflect on the educational implications that media competence currently has in teachinglearning processes. The evolution of ICT and especially mobile technology has led to a communicative and social revolution affecting the educational world; educational contexts are changing and evolving rapidly, they must adapt to sociocultural and technological changes. Three topics are considered in this review study: research on media competence from a neurological standpoint, from the connectivist anthropology one and from the perspective of the teaching-learning process. Three main conclusions are provided: the changes in human communication have encouraged the daily use of a digital language which cannot be ignored in the classrooms, materialized in the need to develop media competence; being essential for this the incorporation of ICT in the school, which also affects the improvement of basic executive functions that enhance learning; finally, teacher training shows a growing trend towards the commitment to respond adequately to the demands of the education of a technological world, also contemplated in the current educational laws and whose complementarity can be interpreted as a guarantee towards an adequate development of media competence in future generations, who must be trained in a fluid and responsible use of ICT and LTK.

Keywords: digital literacy; media competence; digital communication; technological society; educational technology.

Recibido / Received: 09-07-2019

Aceptado / Accepted: 01-01-2020

Publicación en linea / Published online: 30-03-2020

Cómo referenciar este artículo / How to reference this article:

Andreu Ato, N., López-García-Torres, L., \& Saneleuterio, E. (2020). El lenguaje de las tecnologías en la formación de maestros. Tendencias Pedagógicas, 36, pp. 116-128. doi: 10.15366/tp2020.36.09. 


\title{
1. Introducción
}

La llamada sociedad de la información en general, y especialmente las tecnologías en las que se concreta — de la información y la comunicación (TIC) y del aprendizaje y conocimiento (TAC)—, inciden de manera significativa en todos los niveles del proceso educativo. No podemos obviar que vivimos en una sociedad técnica y tecnificada y que es a esta sociedad, con sus particularidades, a la que debe orientarse la educación, en la medida en que esta debe incardinarse en la realidad social inmediata, la que en cada época y contexto concreto nos toca vivir como ciudadanos.

\begin{abstract}
«Alfabetización digital o mediática, competencia digital o mediática, alfabetizaciones múltiples o nuevas alfabetizaciones son los términos más populares que se refieren, con diferentes matices, a los saberes necesarios para convertirse en un ciudadano libre, autónomo y activo en la sociedad contemporánea.» (Alonso-Ferreiro \& Gewerc, 2018, p. 409)
\end{abstract}

Y así, hasta llegar al momento actual, el concepto de educación ha ido evolucionando hacia una concepción absolutamente influenciada por la modernización tecnológica y metodológica, hasta el punto de que hoy en día resulta indiscutible la prioridad asignada a la educación tecnológica en todos los niveles de la educación, formal reglada, no reglada e informal. Nos referimos a la perspectiva amable de los medios, la que posibilita diversas formas de comunicación, el gusto por la pregunta y por descubrir, el conocimiento de nuevos lenguajes (Ramírez \& González, 2016). Una alfabetización necesaria para esta sociedad de la información en la que estamos inmersos, que no puede pasar por alto ni la presencia ni la influencia de los nuevos medios en nuestro desarrollo social, económico y cultural. La educación básica a la que cualquier individuo tiene derecho, en términos de preparación para la vida, es hoy día una «educación para los medios», una educación «mediática» (Gutiérrez, 2015).

Pero nadie discute que el mero conocimiento de los medios tecnológicos, cómo están estructurados y su funcionalidad no logra conducir per se a una verdadera alfabetización digital, es decir, a lo que se conoce como competencia mediática o media proficiency (Aguaded, 2012). Para ello resulta indispensable que exista reflexión crítica ante dichos recursos, por un lado, y, por otro, capacidad para usarlos con soltura y con responsabilidad, en todos los sentidos. En caso contrario, estaríamos ante lo que Santos llama «reducción positivista», que «produce la alucinación de un progreso ascendente en la historia y las sociedades humanas que, aunque técnicamente avancen, pueden hallarse en muchas otras facetas en la Edad de Piedra» (Santos, 2010, p. 74). En definitiva, como sugiere Freire, cada estudiante ha de optar por un equilibrio entre conocer y manejar las nuevas tecnologías y hacer un uso reflexivo, crítico y ético de ellas: «Temo que la curiosidad lograda por una práctica educativa reducida a pura técnica sea una curiosidad castrada que no sobrepasa una posición cientificista ante el mundo» (Freire, 2002, p. 110).

Todo maestro o maestra debe tener siempre presente la necesidad de integrar, dentro de su metodología y estrategias docentes cotidianas, diferentes competencias transversales, y entre ellas nos interesamos por la competencia mediática. Lo que pretende nuestro trabajo es, mediante un estudio de revisión sobre el tema, subrayar cómo esta competencia puede convertirse en factor o elemento diferenciador y potenciador en la formación inicial de docentes. Este objetivo general se concreta en tres objetivos específicos:

- OE1: Explicar el cambio de lenguaje comunicativo que ha sufrido la sociedad debido al desarrollo de las TIC/TAC y de nuevos canales de comunicación digital.

- OE2: Reflexionar sobre las implicaciones educativas en el aprendizaje de la competencia mediática a través del desarrollo de la inteligencia ejecutiva y tecnológica.

- OE3: Determinar las repercusiones de la competencia mediática sobre los docentes en formación, en lo que a la preparación para su futuro profesional se refiere.

\section{La competencia mediática en el ejercicio de la docencia}

El presente estudio de revisión contempla tres resortes: los estudios sobre la competencia mediática desde el ámbito neurológico, desde la antropología conectivista y desde la perspectiva del proceso de enseñanza-aprendizaje.

En cuanto a la evolución del cerebro, es ya clásica la distinción de Prensky $(2001,2015)$ respecto de los conceptos de «nativo digital» e «inmigrante digital» — o, como otros autores los llaman, N-Gen 
(generación en Red) o D-gen (generación digital)—, que desde un punto de vista neurológico se sustenta en su diferente utilización y procesamiento de la información tecnológica: el primero prefiere información ágil, inmediata y visual, y se siente atraído por multitareas y procesos paralelos, así como por métodos de trabajo en red; está habituado a formas lúdicas de aprender y a la satisfacción o recompensa inmediata; el segundo, por el contrario, se muestra más reacio a la utilización de la tecnología y presenta mayor arraigo hacia formas de instrucción textuales y analógicas, debido a que sus procesos de aprendizaje han sido diferentes y su contacto con la tecnología ha requerido de un proceso de adaptación.

En todo caso, unos y otros han acabado convirtiéndose en lo que Sáez Vacas (2011) bautizó como homo digitalis, y posteriormente ha recibido tantas y tantas etiquetas (Fernández \& Fernández, 2016). Y no hay otra opción posible para el ser humano en las sociedades desarrolladas del siglo XXI, pues nuestro cerebro se ha desarrollado de forma que responde al cambio tecnológico, de la misma manera que en épocas anteriores el cerebro humano se adaptó a los avances que nos precedieron (Ansernet \& Magistretti, 2006; Clark \& Chalmers, 1998; García-Carrasco \& Juanes-Méndez, 2013; De la Barrera y Donolo, 2009; Gamo, 2012; Small, 2009). Lasén Díaz añade que «la tecnología, a través de su amplia difusión, personalización y posibilidad de conexión permanentes que crea, contribuye a reconfigurar numerosos aspectos de la vida cotidiana, así como de los procesos de subjetivación y socialización contemporáneos» (Lasén Díaz, 2014, p. 7), hasta llegar a la llamada «generación App» (Gardner y Davis, 2014).

La persona cambia porque las exigencias y necesidades del entorno cambian: hoy somos capaces de memorizar unos patrones y no otros, de manera que generalmente olvidamos datos que no necesitamos recordar porque nuestro omnipresente smartphone lo suple — valgan como ejemplo los números de teléfono de nuestros contactos frecuentes-. El cerebro humano ha evolucionado hacia el uso y la necesidad - e incluso dependencia - de la tecnología. Por ello es crucial que cada docente entienda la importancia de integrar las TIC dentro de sus planificaciones educativas, no solo en actividades en las que se aprenden a usar los entornos digitales, sino como medio para lograr los fines educativos (Prensky, 2015).

En esta línea, Marina (2012) establece la necesidad de generar espacios de aprendizaje para las inteligencias generadora y ejecutiva, así como de facilitar la inclusión de las tecnologías en los procesos de aprendizaje de una forma más natural; si los cerebros actuales están preparados para la multitarea que plantean las TIC y las TAC, la educación debería aprovechar ese potencial para optimizarlo y encauzarlo hacia fines beneficiosos para el desarrollo adecuado del alumnado. Es más, como señala Gutiérrez (2015), sería un error considerar dos realidades independientes de partida: la tecnología y la educación, y, por lo tanto, considerar una educación sin tecnología.

En este contexto, existen estudios que ya intentaban cuantificar este mundo hiperconectado por la tecnología. Ya hace más de cinco años, según los datos de Gimeno (2013), el 69,3\% de la población utilizaba WhatsApp a diario, el 46,5 \% Facebook, el 34,2 \% Skype y el 29,5 \% Live Messenger/MSN Messenger. En comparativa, podemos comprobar la evolución en el estudio anual de uso de redes sociales elaborado por IAB (2019), según el cual, en España, la población entre 16 y 65 años manifiesta que el $85 \%$ utiliza las redes sociales a diario. De estos, el $40 \%$ del alumnado de secundaria y el $46 \%$ de estudiantes universitarios usa las redes. Así, la red más usada es WhatsApp, con un $88 \%$, declarando que es usada diariamente por un $97 \%$, seguida de Facebook con un $87 \%$, usada a diario por un $73 \%$, y en tercer lugar YouTube, con un $68 \%$ y un uso diario del $70 \%$. Además, es llamativo mencionar que el $65 \%$ del uso que se realiza de estas plataformas tiene como objetivo comunicarse y relacionarse socialmente, siendo el móvil con acceso a internet el dispositivo tecnológico más usado.

Lo que resulta indiscutible es que la sociedad en la que vivimos está demandando cambios desde la base teórica y práctica de la educación para ajustarse a la nueva realidad, frente al carácter casi residual de precauciones alarmantes, de inspiración platónica, que conciben el conocimiento epistemológico como algo reservado a otro tipo de praxis (Martín-Gordillo \& González-Galbarte, 2002). Así, es natural que haya surgido la tendencia tecnoantropóloga para explicar la relación entre tecnología, sociedad, bienestar y cultura en general, así como la raíz tecnológica de los diseños culturales, su impacto y su difusión (Colobrans et al., 2012).

Esta concepción del ser humano aboga por pasar de una visión constructivista del aprendizaje humano - maestro o maestra como guía para que cada estudiante indague, busque información, reflexione, establezca objetivos y construya nuevos contenidos para así poder asimilar y generalizar 
otros- a una nueva perspectiva psicológica denominada conectivismo, que responde a la conectividad de entorno virtual (Canales \& Marquès, 2007; Siemens, 2004). Para Manzano (2013), en este nuevo contexto educativo, los agentes educativos se convierten en "prosumidores», es decir, productores de nuevos conocimientos que se difunden y quedan al alcance del público.

Así, se entiende que la alimentación y mantenimiento de las conexiones es necesaria para facilitar el aprendizaje continuo: la habilidad de ver conexiones entre áreas, ideas y conceptos es una habilidad clave, necesaria para la actualización de conocimiento. La toma de decisiones, por tanto, en el uso de la tecnología, requiere en sí misma un proceso de aprendizaje que deja de ser individual e interno para globalizarse (Reig Hernández, 2010), siendo la misión del educador la de guiar por esos nuevos entornos virtuales, donde se aprende de los otros y con los otros.

Desde la perspectiva de Delors (1996), se ha evolucionado a una concepción conectivista del conocimiento que, según Siemens (2010), implica hoy en día «saber sobre x», «saber hacer x», «saber ser $\mathrm{x}$, «saber dónde $\mathrm{x} » \mathrm{y}$ «saber transformar $\mathrm{x}$ » para llegar, en este último estadio, a un nivel profundo del conocimiento, comprendiendo sus causas para ser capaces de innovar. Actualmente, empero, se busca más la capacidad de aprender a crear, propia de la Generación Einstein enunciada por Boschma (2008, p. 98):

«la primera generación de jóvenes desde la Segunda Guerra Mundial que se identifica por su inteligencia y por características positivas: sociabilidad, cooperación, inteligencia, implicación, entre otras. Conoce perfectamente los medios de información: entiende los anuncios, la publicidad y la comunicación. Desea hacerlo todo anywhere, anytime, anyplace.»

Así Núñez, García y Hermida (2012, p. 8) especifican que los miembros de esta generación «no solo reciben y producen contenidos digitales, sino que forman parte de la retícula que vela por la gestión de la información en la red, así como de las acciones de los internautas en las interrelaciones entre los distintos medios», convirtiéndose por tanto en gestores críticos de la información. Además, entre otras cosas, el conectivismo exige de la persona emprendimiento ya que el conocimiento es cambiante y el diálogo, incluido el digital, potencia el aprendizaje (Ledesma Ayora, 2015).

Todo ello requiere un proceso de educación en comunicación que la UNESCO (2002) definió como una disciplina autónoma que pretende enseñar y aprender sobre los medios de comunicación, incluidos los tecnológicos, produciendo un análisis crítico y de producción creativa, provocando un espíritu de comunidad y de responsabilidad social, así como autonomía personal. En efecto, según la Recomendación 2006/962/CE, sobre las competencias clave para el aprendizaje permanente, la competencia digital implicaba el uso crítico y seguro de las TIC en ámbitos laborales, académicos y cotidianos, tanto para comunicarse como para realizar usos diversos de la información (buscar, seleccionar, analizar, producir, presentar, intercambiar...) mediante el uso de dispositivos electrónicos y conexión a internet.

Avanzando en el siglo XXI, la incorporación de la tecnología a la escuela ha pasado desde la alfabetización con TIC a su concepción y uso de las como herramienta de aprendizaje. En España, esto se refleja en la evolución legislativa de la incorporación de las TIC en la enseñanza (Manzano, 2013). Así, la Ley Orgánica 8/2013, de 9 de diciembre, para la mejora de la calidad educativa (LOMCE) y sus diferentes concreciones curriculares — Real Decreto 126/2014; Orden ECD/65/2015—, así como los correspondientes decretos autonómicos establecen que la competencia digital, como transversal, debe impregnar todas las áreas.

El problema viene cuando la normativa choca con la realidad de las aulas. El Estudio Internacional sobre la Enseñanza y el Aprendizaje, denominado TALIS (OCDE, 2009; 2014), recogió que el profesorado autopercibe, por lo general, que no se siente preparado adecuadamente para una buena integración de las TIC en sus clases, si bien otros estudios muestran diferencias según las comunidades autónomas en cuanto al uso de libros, mochilas y aulas digitales, pizarras interactivas, etc., frecuentemente vinculadas a la mayor o menor partida presupuestaria destinada a ello (Area et al., 2014).

Finalmente, si exploramos la literatura que intenta responder a por qué es necesario que la competencia mediática y, sobre todo, las TIC sean utilizadas como herramienta en los procesos de enseñanza-aprendizaje, encontramos referentes para todas las etapas educativas, siendo positivo el efecto del uso de ordenadores, por ejemplo, en el rendimiento académico, especialmente en las clases sociales más desfavorecidas (OCDE, 2013). Considerando estos beneficios, el uso de las TIC en las aulas viene siendo considerado como un factor de inclusión y equidad educativa (OCDE, 2013). Aparte de 
su potencial innovador para reducir el fracaso escolar, Marquès (2012) añade dos razones más para usar las TIC en educación: la absoluta necesidad de la alfabetización digital y la productividad y ventajas comunicativas que proporcionan.

La alfabetización digital es definida por Casado como:

«el proceso de adquisición de los conocimientos necesarios para conocer y utilizar adecuadamente las tecnologías y poder responder críticamente a los estímulos y exigencias de un entorno informacional cada vez más complejo, con variedad y multiplicidad de fuentes, medios de comunicación y servicios.» (Casado, 2006, p. 68)

Según autores como Vivancos (2008) y Acosta (2010), esta competencia incluye los tres tipos de alfabetización recogidos por la Directiva 2010/13/UE, relativa a la prestación de servicios de comunicación audiovisual:

- La alfabetización audiovisual, que se define como «la comunicación mediática que abarca las habilidades, los conocimientos y las capacidades de comprensión que permiten a los consumidores utilizar con eficacia los medios» (Vivancos, 2008, p. 33).

- La alfabetización informacional, que es «la adquisición de habilidades de tratamiento de la información: búsqueda, selección, procesamiento y comunicación para transformarla en conocimiento aplicando tecnologías apropiadas» (Vivancos, 2008, p. 35).

- La alfabetización en TIC o digital provocada a partir de la aparición del ordenador y de la necesidad de aprender su uso y, sobre todo, su usabilidad (Acosta, 2010).

Vivancos (2008) y Acosta (2010) sistematizan en cinco las dimensiones de las TIC según el MECD:

- Cognitiva: facilitan la construcción de conocimiento individual y compartido.

- Comunicacional: requieren argumentar la selección de fuentes, contenidos y uso de herramientas de comunicación.

- Colaborativa: facilitan el desarrollo de habilidades para el trabajo en equipo.

- Axiológica-ética: desarrollan la ciudadanía digital y el uso seguro y respetuoso de recursos y redes.

- Tecnología instrumental: capacitan para el uso de la tecnología en general.

Para Area y Ribeiro (2012), explicar el modelo de alfabetización digital o de desarrollo de la competencia digital debe ser un proyecto que contemple a su vez la obtención de subcompetencias TIC a partir de la consecución de las siguientes:

- Competencia instrumental (conocimiento práctico, habilidades y estrategias para el uso de hardware y software cambiante).

- Competencia cognitivo-intelectual (capacidad de crear nuevos contenidos digitales a partir de la búsqueda, análisis, interpretación, etc.), que Carneiro (2009) vincula a la autorregulación del aprendizaje.

- Competencia socio-comunicacional (habilidades sociales, trabajo colaborativo, empatía, etc.) (Padhila, 2009).

- Competencia axiológica (conciencia de que las TIC nunca son neutrales, capacidad crítica para detectar la intencionalidad cultural, política, social, etc.).

- Competencia emocional (gestión adecuada de las emociones también en entornos digitales).

Urueña (2016, p. 219), por su parte, argumenta así la necesidad de la integración de las TIC: «viene dada por lograr las competencias necesarias para el correcto desenvolvimiento en el medio que nos rodea (sociedad tecnológica y del conocimiento) y por fomentar una cultura y ethos tecnológico que apoye una ciudadanía crítica y con ímpetu participativo y comprensivo en lo que al uso, diseño e implementación de las tecnologías se refiere.»

Para Marquès (2012), lo importante será el diseño y la planificación del uso de las TIC en el aula, ya que es determinante cómo utiliza dicha tecnología cada docente: con papel y tiza puede ser muy capaz de desarrollar su docencia, pero el uso que desarrolle con las herramientas virtuales será lo que 
marque la diferencia y la posibilidad de que sus estudiantes desarrollen simultáneamente la competencia digital, además de que muchos estudios demuestran su potencial para motivar - siempre que la calidad de la experiencia esté garantizada (Area, 2010; Espósito \& Manzano, 2012) - e incluso multiplicar el aprendizaje y prevenir o afrontar dificultades (Bernardo, Bernardo y Herrero, 2005; Coll, Onrubia, \& Mauri; 2007; More, 2008; Orit \& Shrieber, 2004; Ressa, 2010), a pesar de sus limitaciones (García-García, 2006) y ciertas inseguridades en las que hay que trabajar, sobre todo en cuanto a protección de datos (De Pablos, 2010): la enseñanza digital debe basarse en un modelo educativo para la sociedad digital, educación como servicio a la persona, no al mercado ni a la competitividad (Murillo García, 2010).

Por supuesto, el uso de las TIC en el aprendizaje es positivo en todas las áreas y etapas educativas, y de eso es consciente el Espacio Europeo de Educación Superior (Ferro, Martínez y Otero, 2009), en la medida en que rompe con la espacialidad de la enseñanza, permite usar el ciberespacio como marco de enseñanza y herramienta básica para el docente, consigue la participación activa de cada estudiante en su proceso de aprendizaje, no solo desde la recepción, sino sobre todo desde su autoría, incrementando sus posibilidades formativas. Además, esto es especialmente relevante en las carreras de Magisterio, pues si las competencias docentes en la era de internet son diferentes a las que se han requerido en épocas anteriores (Marquès, 2014), también la formación universitaria de estos profesionales ha de ser diferente y prever un desarrollo adecuado, entre otras, de su propia competencia mediática (Liesa Orús, Vázquez Toledo, \& Lloret Gazo, 2016).

Valverde Berrocoso, Fernández Sánchez y Revuelta Domínguez (2013) encuestaron a docentes de Infantil, Primaria y Secundaria sobre sus estados emocionales y bienestar subjetivo con el uso de las TIC en sus procesos educativos y comprobaron que más de la mitad considera un deber y una responsabilidad docente el uso de las TIC en el aula, además de porque son imprescindibles para desarrollar la competencia digital de sus alumnos, porque rompen la monotonía en la enseñanza y ayudan a evitar el aburrimiento. A pesar de las posibles pérdidas de tiempo que ocasiona la pizarra digital interactiva (PDI) en las aulas debido a los eventuales problemas técnicos, un estudio anterior (Domingo y Marquès, 2011) ya mostraba que el $91 \%$ del profesorado consideraba que las TIC mejoran tanto los procesos como los aprendizajes, aunque para el $46 \%$ ello no tiene relación directa con el incremento del rendimiento académico. Para Marquès (2013), la causa de esto sería que los sistemas de evaluación no han evolucionado para medir las capacidades competenciales del alumnado.

En el ámbito universitario, Morales, Trujillo y Raso (2015) mostraron que, aunque se considera indiscutible la utilización de las TIC en los procesos de enseñanza-aprendizaje, el $40 \%$ de los alumnos manifestaron que sus profesores las utilizan deficientemente y que no se preocupan por asegurar un papel más activo al estudiante en su uso. Por su parte, Gallego et al. (2016) analizaron una experiencia de aprendizaje ubicuo en el Grado en Educación Primaria y mostraron que el alumnado, a pesar de no conocer bien las herramientas digitales previamente, adquiría una competencia digital muy adecuada en su uso y aprendizaje con ellas, y percibieron que las actividades realizadas eran transferibles a otras etapas educativas.

\section{Implicaciones educativas}

El sistema educativo español, al igual que las regulaciones actuales de la educación en casi todos los países, está plagado de referencias a la competencia digital como competencia básica dentro del proceso educativo. En concreto, en el currículum de las etapas educativas en España viene expuesta de forma explícita, ya sea como elemento transversal del currículum o como competencia básica que se ha alcanzar en la etapa.

La UNESCO, en 2014, manifestaba que son más los dispositivos digitales existentes que habitantes hay en el planeta. Ello implica que los niños y adolescentes en su mayoría tienen acceso muy temprano a las TIC desde sus hogares: precisamente la competencia digital se adquiere más por convivencia con las TIC en contextos informales que por un adecuado desarrollo en la escuela que potencie gradual y progresivamente su adquisición (Pérez-Escoda, Castro-Zubizarreta, \& Fandos, 2016), y este hecho hace imperiosa la necesidad de que desde la educación reglada se garantice una adecuada competencia mediática.

Si nos centramos en la Educación Superior, la realidad tecnológica tiene un alto impacto en la cultura universitaria debido a que implica el acceso generalizado de los estudiantes, la necesidad de formación continua, una mayor exigencia de calidad y flexibilidad, la transformación de la función 
docente... (Falco, 2017). Aunque la brecha generacional marca una barrera dentro de los procesos de enseñanza-aprendizaje exigidos en la universidad (Quicios, 2015), la docencia deberá trabajar por suplir las carencias de los «inmigrantes digitales» en el uso de las TIC para conseguir una formación de calidad que responda a los requerimientos del mundo laboral donde potencialmente se puede incorporar cada estudiante que egresa. Esto quiere decir que la formación universitaria, y especialmente la de futuros maestros y profesores, debe conseguir que todos los alumnos estén formados en el uso instrumental, pero sobre todo profesional, de las TIC: su presencia en las aulas no solo contribuirá a la mejora de la competencia mediática de los niños y niñas que les sean encomendados, sino que también aumentará su interés y motivación, factores claves en el aprendizaje. Porque aunque algunas teorías sobre la motivación en el aprendizaje se plantean como independientes del entorno en el cual se desarrolla el proceso formativo (presencial, TIC, virtual...), otras plantean una estrecha relación (Gómez García, Ferrer, \& De la Herrán, 2015).

De manera complementaria a todo lo expuesto, hemos de considerar que otra cuestión clave en el aprendizaje de la competencia mediática es la formación axiológica. Area (2008, p. 68) no entiende la alfabetización en la cultura digital separada del «cultivo y desarrollo de actitudes y valores que otorguen sentido y significado moral, ideológico y político a las acciones desarrolladas con las tecnologías». Debemos considerar la ética de la tecnología como una parte indispensable del proceso de formación integral de los estudiantes, encaminándolos a ser buenos maestros y, lo que es más importante, buenos ciudadanos capaces de abrirse camino a través de una sociedad tecnológica cada vez más avanzada. En este sentido proponemos como referencia obligada el catálogo de virtudes de la profesora Shannon Vallor, de la Universidad de Santa Clara, en California, que consideramos imprescindibles en el proceso de formación de los docentes del futuro. Vallor (2016) identifica doce virtudes tecno-morales, que estima como hábitos y prácticas para educar personas valiosas en el contexto de la sociedad contemporánea: honestidad, autocontrol, humildad, justicia, coraje, empatía, cuidado, civilidad, flexibilidad, perspectiva, magnanimidad y sabiduría tecno-moral.

Esa doble —y necesaria - vertiente de educar con medios y educar sobre los medios sigue poniendo de manifiesto la carencia de la segunda en las instituciones educativas. Mientras que la tecnología educativa está incluida en los planes de formación del profesorado, el estudio de los medios y la educación para su utilización sigue luchando sin demasiado éxito por hacerse un hueco en los currículos (Gutiérrez \& Tyner, 2012).

Por suerte, un estudio reciente realizado por Álvarez-Arregui et al. (2017) con población universitaria española y mexicana, entre los que había futuros maestros, pone de manifiesto que la mayoría de los estudiantes valora positivamente la importancia de la competencia mediática en la sociedad y la gestión de información, a la vez que reconoce sus riesgos y se inclinan positivamente por el consumo ético y responsable.

Por último, debemos entender las TIC como un instrumento de cambio que ayudará al desarrollo de los procesos de enseñanza-aprendizaje, pero no como un cambio de todos los procesos de enseñanza que conocemos. Las TIC son una herramienta más en el aula para desarrollar procesos de comunicación educativa, planificación docente, organización del aula, búsqueda de información, etc. Se trata de una educación mediática: «que supere la visión excesivamente tecnológica e instrumental que, fruto de las modas y los avances tecnológicos, a menudo ha confundido a políticos, administradores y sociedad en general y ha distorsionado e ignorado las inherentes características y cualidades que los medios tienen de cara a la educación.» (Aguaded, 2012, p. 260).

Asimismo, Marquès (2012, p. 12) nos recuerda que, dentro del proceso de enseñanza-aprendizaje, «lo relevante debe ser siempre lo educativo, no lo tecnológico». Expone que la figura docente es la que será relevante como mejora de los aprendizajes de sus estudiantes, por medio de un diseño didáctico que integre las TIC como innovación de su enseñanza.

Nosotras sugerimos el modelo para la formación del profesorado en TIC y medios propuesto por Gutiérrez (2018), que parte de la precisión de conceptos para no caer en reduccionismos, y plantea una intervención concreta en torno a cinco bloques temáticos: 1) alfabetización múltiple en la era digital; 2) medios y entornos digitales como agentes de educación informal; 3) integración curricular de los medios y tecnologías digitales como recursos didácticos; 4) integración curricular de TIC y medios como objeto de estudio: educación mediática en la enseñanza formal; 5) la educación mediática en la enseñanza universitaria y en la formación inicial del profesorado.

Y no perdemos de vista la idea expuesta acerca del valor de los maestros y maestras como factor esencial en los procesos de aprendizaje, con y sin TIC. Porque no hemos de olvidar que, al hablar de 
docentes de calidad no aludimos a quienes usan o no las TIC en el aula, ni a quienes consiguen los mejores resultados, sino a quienes saben entender las necesidades de aprendizaje de sus alumnos, quienes buscan interesarlos, motivarlos y, sobre todo, como fin último, intentar participar en la felicidad de cada estudiante.

\section{Conclusiones}

Si recapitulamos lo expuesto hasta el momento y lo relacionamos con los objetivos explicitados al principio y la literatura cotejada, confirmamos la influencia de la competencia mediática en el aprendizaje, ya sea de forma directa y explícita o de forma transversal, para llegar a conseguir los propósitos de la educación en la sociedad de la información.

En primer lugar, respecto al cambio de lenguaje comunicativo que ha sufrido la sociedad debido al desarrollo de las TIC y las TAC y, en concreto, de nuevos canales de comunicación digital (OE1), podemos afirmar que la habituación a la comunicación según las reglas discursivas de las redes sociales, el uso de aplicaciones móviles, etc., fomentan cierto relegamiento del lenguaje textual y presencial a determinados ámbitos de uso, en favor de un lenguaje sobre todo digital, icónico y con abundante uso de emoticonos en usos escritos que trascienden en WhatsApp o Messenger para conquistar correos electrónicos e incluso textos manuscritos de diversa índole.

Respecto al OE2, mediante el que pretendíamos reflexionar sobre las implicaciones en el aprendizaje de la competencia mediática a través del desarrollo de la inteligencia ejecutiva y tecnológica, los resultados nos llevan a concluir que las TIC pueden convertirse en una herramienta que genere en el alumnado niveles superiores de percepción, atención, comunicación, etc., funciones ejecutivas básicas para la mejora de los aprendizajes. El conocimiento se crea a partir de la interacción y se estructura en varias fases: cocreación, distribución, comunicación de ideas clave, personalización e implementación (Siemens, 2010). Este proceso exige un nivel de autorregulación cognitiva elevado para que los aprendizajes se produzcan de forma óptima. Por ello es crucial que el sistema educativo integre las TIC dentro de las planificaciones educativas, no solo en actividades en las que aprendamos a usar los entornos digitales, sino como un elemento más en el aula, que al final, por la cotidianidad haga desaparecer en parte lo novedoso de las TIC y así el alumnado «aprenda a aprender» con las TIC (Prensky, 2015).

El desarrollo de la competencia mediática, prevista como parte de las competencias básicas en las legislaciones educativas, implica contemplar la alfabetización digital en las aulas (Area \& Ribeiro, 2012; Vivancos, 2008), proceso que requiere una planificación que asegure el uso estratégico de la tecnología, basado en objetivos y metas de aprendizaje (Rioseco \& Roig, 2014) y que pase por el logro de las competencias instrumentales, cognitivas e intelectuales, socio-comunicacionales, axiológicas y emocionales.

La literatura aportada en la discusión nos permite concluir, también, que el uso de las TIC en el aula mejora el aprendizaje de los alumnos - y, por ende, su rendimiento académico- siempre que se ajusten los procedimientos de evaluación a la nueva realidad y demanda educativa, además de convertirse en elemento motivador del aprendizaje e impulsor del proceso de aprender a aprender (Area, 2010; Bernardo, Bernardo, \& Herrero, 2005; Coll, Onrubia, \& Mauri, 2007; Domingo \& Marquès, 2011; Ferro, Martínez, \& Otero, 2009; Morales, Trujillo, \& Raso, 2015; More, 2008; Orit \& Shrieber, 2004; Ressa, 2010; Valverde Berrocoso, Fernández Sánchez, \& Revuelta Domínguez, 2013).

Finalmente, por lo que se refiere al $\mathrm{OE} 3$, que atendía las repercusiones de la competencia mediática sobre los docentes en formación, concluimos que tanto el profesorado como el alumnado universitario son conscientes de su responsabilidad de educar con y sobre las TIC y las TAC, y consideran que ello resulta imprescindible para desarrollar la competencia mediática, básica para desenvolverse en el mundo actual y futuro.

Además, la mayoría de los estudiantes de Magisterio se adapta fácilmente a las innovaciones tecnológicas que se les proponen en la universidad, e incluso suelen percibir que ciertos planteamientos de las actividades realizadas en el aula universitaria pueden ser transferibles a las etapas educativas donde se incorporarán como docentes. Son conscientes de que la sociedad del siglo XXI exige visualizar el mundo educativo desde perspectivas diversas a las planteadas hasta hace poco; es por este 
motivo por el que sienten que los modelos educativos y, sobre todo, las escuelas deben responder a las necesidades y demandas cambiantes.

En definitiva, a la par de la preocupación por la competencia mediática reflejada en la legislación educativa, las vías adecuadas de su concreción curricular en la praxis quedan garantizadas paulatinamente por las tendencias docentes de la escuela hacia la toma de conciencia, desde la formación de las nuevas generaciones de profesorado, de la necesidad de un consumo responsable y de una instrucción reglada en este sentido, no abandonada a lo azaroso del hogar.

\section{Referencias}

Acosta, L. M. (2010). El tratamiento de la información y competencia digital (TICD) en la enseñanzaaprendizaje de la historia del bachillerato. Catharum. Revista de Ciencias y Humanidades del Instituto de Estudios Hispánicos de Canarias, 11, pp. 57-67. Recuperado de https://dialnet.unirioja.es/servlet/articulo? codigo $=3769780$.

Aguaded, I. (2012). La competencia mediática, una acción educativa inaplazable. Comunicar, 39, pp. 7 8. doi: 10.3916/C39-2012-01-01

Alonso-Ferreiro, A., y Gewerc, A. (2018). Alfabetización mediática en la escuela primaria. Estudio de caso en Galicia. Revista Complutense de Educación, 29(2), pp. 407-422. doi: 10.5209/RCED.52698

Álvarez-Arregui, E., Rodríguez-Martín, A., Madrigal-Maldonado, R., Grossi-Sampedro, B., \& Arreguit, X. (2017). Ecosistemas de formación y competencia mediática: Valoración internacional sobre su implementación en la educación superior. Comunicar, 51, pp. 105-114. doi: 10.3916/C512017-10

Ansernet, F., \& Magistretti, P. (2006). A cada cual su cerebro. Plasticidad neuronal e inconsciente. Buenos Aires: Katz Editores. doi: https://doi.org/10.2307/j.ctvnp0kn5.

Area, M. (2010). El proceso de integración y uso pedagógico de las TIC en los centros educativos. Un estudio de casos. Revista de Educación, 352(2), pp. 77-97. Recuperado de http://www.revistaeducacion.educacion.es/re352/re352 04.pdf.

Area, M., Alonso, C., Correa, J. M., Del Moral, M. E., De Pablos, J., Paredes, J., Peirats, J., Sanabria, A. L., San Martín, A., \& Valverde Berrocoso, J. (2014). Las políticas educativas TIC en España después del Programa Escuela 2.0: las tendencias que emergen. RELATEC. Revista Latinoamericana de Tecnología Educativa, 13(2), pp. 11-33. doi: 10.17398/1695-288X.13.2.11

Area, M., Gros, B., \& Marzal, M. A. (2008). Alfabetizaciones y tecnologías de la información y la comunicación. Madrid: Síntesis.

Area, M., \& Ribeiro, M. T. (2012). De lo sólido a lo líquido: Las nuevas alfabetizaciones ante los cambios culturales de la Web 2.0. Comunicar, 38, pp. 13-20. doi: 10.3916/C38-2012-02-01

Bernardo, I., Bernardo, A., \& Herrero, J. (2005). Nuevas tecnologías y educación especial. Psicothema, 17(1), pp. 64-70. Recuperado de http://www.psicothema.com/pdf/3065.pdf

Boschma, J. (2008). Generación Einstein: más listos, más rápidos y más sociables. Barcelona: Gestión 2000.

Canales, R., \& Marquès, P. (2007). Factores de buenas prácticas educativas con apoyo de las TIC: análisis de su presencia en tres centros educativos. Educar, 39, pp. 115-133. doi: 10.5565/rev/educar.164

Carneiro, R. (2009). Las TIC y los nuevos paradigmas educativos: la transformación de la escuela en una sociedad que se trasforma. En R. Carneiro, J. C. Toscano y T. Díaz (coords.), Los desafíos de las TIC para el cambio educativo (pp. 15-28). Madrid: Fundación Santillana.

Casado, R. (Dir.) (2006). Claves de la alfabetización digital. Madrid: Fundación Telefónica. Recuperado de http://www.anamorenoromero.net/documentos/Anexo3 claves alfabetiz digital.pdf.

Clark, A., \& Chalmers, D. (1998). The extended mind. Analysis, 58(1), pp. 7-19. doi: 10.1093/analys/58.1.7

Coll, C., Onrubia, J., \& Mauri, T. (2007). Tecnología y prácticas pedagógicas: las TIC como instrumentos de mediación de la actividad conjunta de profesores y estudiantes. Anuario de Psicología, 38(3), pp. 377-400. Recuperado de http://www.redalyc.org/articulo.oa?id=97017407003.

Colobrans, J., Serra, A., Faura, R., Bezos, C., \& Martin, I. (2012). La tecno-antropología. Revista de Antropologia Experimental, 12(9), pp. 137-146. Recuperado de https://revistaselectronicas.ujaen.es/index.php/rae/article/view/1909/1659. 
De la Barrera, M. L., \& Donolo, D. (2009). Neurociencias y su importancia en contextos de aprendizaje. Revista Digital Universitaria, 4(10). Recuperado de http://www.revista.unam.mx/vol.10/num4/art20/art20.pdf.

De Pablos, J. (2010). Universidad y sociedad del conocimiento. Las competencias informacionales y digitales. RUSC. Revista de Universidad y Sociedad del Conocimiento, 7(2), pp. 6-16. Recuperado de http://hdl.handle.net/10609/2603.

Delors, J. (1996). La educación encierra un tesoro. Informe a la UNESCO de la comisión Internacional sobre la Educación para el siglo XXI. Madrid: Santillana Unesco.

Directiva 2010/13/UE del Parlamento Europeo y del Consejo, de 10 de marzo, sobre la coordinación de determinadas disposiciones legales, reglamentarias y administrativas de los Estados miembros relativas a la prestación de servicios de comunicación audiovisual. Diario Oficial de la Unión Europea, L 95/1, de 15 de abril de 2010. Recuperado de http://www.minetad.gob.es/telecomunicaciones/mediosaudiovisuales/Documents/Directiva Servicios.pdf.

Domingo, M., \& Marquès, P. (2011). Aulas 2.0 y uso de las TIC en la práctica docente. Comunicar, 37, pp. 169-175. doi: 10.3916/C37-2011-03-09

Expósito, J., \& Manzano, B. (2012). New digital learning models in educational process. Acta Humanitá, 4.

Falco, M. (2017). Reconsiderando las prácticas educativas: TICs en el proceso de enseñanza-aprendizaje. Tendencias Pedagógicas, 29, pp. 59-76. Recuperado de https://revistas.uam.es/tendenciaspedagogicas/article/view/7084/7450. doi: 10.15366/tp2017.29.002.

Fernández, F. J., \& Fernández, M. J. (2016). Los docentes de la Generación Z y sus competencias digitales. Comunicar, 46, pp. 97-105. doi: 10.3916/C46-2016-10

Ferro, C., Martínez, A. I., \& Otero, M. C. (2009). Ventajas del uso de las TICs en el proceso de enseñanza-aprendizaje desde la óptica de los docentes universitarios españoles. EDUTEC. Revista Electrónica de Tecnología educativa, 29, pp. 1-12. doi: 10.21556/edutec.2009.29.451

Freire, P. (2002). A la sombra de este árbol. Barcelona: El Roure.

Gallego-Lema, V., Muñoz-Cristóbal, J. A., Arribas-Cubero, H. F., \& Rubia-Avi, B. (2016). Aprendizaje ubicuo: un proceso formativo en educación física en el medio natural. RELATEC Revista Latinoamericana de Tecnología Educativa, 15(1), pp. 59-73. doi: 10.17398/1695-288X.15.1.59

Gamo, J. R. (2012). La neuropsicología aplicada a las ciencias de la educación: una propuesta que tiene como objetivo acercar al diálogo pedagogía/didáctica, el conocimiento de las neurociencias y la incorporación de las tecnologías como herramientas didácticas válidas en el proceso de enseñanza-aprendizaje. En J. Navarro, M. T. Fernández, F. J. Soto, \& F. Tortosa (Coords.), Respuestas flexibles en contextos educativos diversos (pp. 1-15). Murcia: Consejería de Educación, Formación y Empleo de la Región de Murcia. Recuperado de http://diversidad.murciaeduca.es/publicaciones/dea2012/docs/jrgamo.pdf.

García-Carrasco, J., \& Juanes-Méndez, J. A. (2013). El cerebro y las TIC. Teoría de la Educación, Educación y Cultura en la Sociedad de la Información, 14(2), pp. 42-84. Recuperado de http://www.redalyc.org/articulo.oa?id=201028055003.

García-García, F. (2006). Contenidos educativos digitales: Construyendo la Sociedad del Conocimiento. Revista de Tecnologias de la Información y la Comunicación Educativa, 6, pp. 1-29. Recuperado de http://reddigital.cnice.mec.es/6/Articulos/pdf/Articulos 1.pdf.

Gardner, H., \& Davis, K. (2014). La generación APP. Barcelona: Paidós.

Gimeno, M. (Dir.) (2013). eEspaña. Informe anual 2013 sobre el desarrollo de la sociedad de la información en España. Madrid: Fundación Orange. Recuperado de http://www.proyectosfundacionorange.es/docs/eEspana 2013 web.pdf.

Gómez García, M., Ferrer, R., \& De la Herrán, A. (2015). Las redes sociales verticales en los sistemas formales de formación inicial de docentes. Revista Complutense de Educación, 26(núm. Especial), pp. 215-232. doi: $10.5209 /$ rev RCED.2015.v26.46330

Gutiérrez, A. (2015). Educación y nuevos medios: ¿convergencias ignoradas o previstas? Redes.com. Revista de estudios para el desarrollo social de la Comunicación, 12, pp. 19-25.

Gutiérrez, A. (2018). Educación Mediática y su Didáctica. Una Propuesta para la Formación del Profesorado en TIC y Medios. Revista Interuniversitaria de Formación del Profesorado, 91(32.1), pp. 15-27. Recuperado de https://dialnet.unirioja.es/servlet/articulo? codigo $=6441409$.

Gutiérrez, A., \& Tyner, K. (2012). Educación para los medios, alfabetización mediática y competencia digital. Comunicar, 38, pp. 31-39. doi: 10.3916/C38-2012-02-03 
IAB Spain (2019). Estudio anual de Redes Sociales 2019. Recuperado de https://iabspain.es/wp-content/uploads/estudio-anual-redes-sociales-iab-spain-019 vreducida.pdf.

Lasén Díaz, A. (2014). Introducción: Las mediaciones digitales de la educación sentimental de los y las jóvenes. En I. Megías Quirós, \& E. Rodríguez San Julián (Coords.) Jóvenes y comunicación: la impronta de lo virtual (pp. 7-16). Madrid: Fundación de Ayuda contra la Drogadicción. Recuperado de https://es.scribd.com/document/217402107/jovenes-y-comunicacion-La-impronta-de-lovirtual-1-pdf.

Ledesma Ayora, M. A. (2015). Del conductismo, cognitivismo y constructivismo al conectivismo para la Educación. Quito: Editorial Jurídica Ecuador. Recuperado de https://app.box.com/s/uvgbgui08ey57e0beok154lhe792hymh.

Ley Orgánica 8/2013, de 9 de diciembre, para la mejora de la calidad educativa. Boletín Oficial del Estado, 295, de 10 de diciembre. Recuperado de https://www.boe.es/boe/dias/2013/12/10/pdfs/BOE-A-2013-12886.pdf.

Liesa Orús, M., Vázquez Toledo, S., \& Lloret Gazo, J. (2016). Identificación de las fortalezas y debilidades de la competencia digital en el uso de aplicaciones de internet del alumno de primer curso del Grado de Magisterio. Revista Complutense de Educación, 27(2), pp. 845-862. doi: 10.5209/rev RCED.2016.v27.n2.48409

Manzano García, I. (2013). Escuela TIC 2.0: Aprendizaje del alumnado de Primaria en su contexto educativo y sociofamiliar. Granada: Editorial Universidad de Granada. Recuperado de http://digibug.ugr.es/bitstream/10481/24564/1/21881698.pdf.

Marquès, P. (2012). Impacto de las TIC en la educación: funciones y limitaciones. 3C TIC: Cuadernos de desarrollo aplicados a las TIC. Recuperado de https://www.3ciencias.com/wp-content/uploads/2013/01/impacto-de-las-tic.pdf.

Marquès, P. (2013). Investigación: uso didáctico de los contenidos digitales. 3C TIC: Cuadernos de desarrollo aplicados a las TIC. Recuperado de https://www.3ciencias.com/wp-content/uploads/2013/04/EDUCALINE-PERE-MARQUES.pdf.

Marquès, P. (2014). Competencias docentes en la era de Internet. Recuperado de https://es.slideshare.net/peremarques/competencias-docentes-en-la-era-internet.

Marina, J. A. (2012). Neurociencia y Educación. Participación Educativa, 1(1), pp. 6-14. Recuperado de http://ntic.educacion.es/cee/revista/pdfs/RPE DIC2012 02Marina.pdf.

Martín Gordillo, M., \& González-Galbarte, J. C. (2002). Reflexiones sobre la educación tecnológica desde el enfoque CTS. Revista Iberoamericana de Educación, 28(1). Recuperado de https://rieoei.org/historico/documentos/rie28a01.htm.

MECD (2016). Competencias clave: competencia digital. Recuperado de http://www.mecd.gob.es/educacion-mecd $/ \mathrm{mc} /$ lomce/el-curriculo/curriculo-primaria-eso-bachillerato/competencias-clave/digital.html.

Morales, M., Trujillo, J. M., \& Raso F. (2015). Percepciones acerca de la integración de las TIC en el proceso de enseñanza-aprendizaje de la universidad. Pixel-Bit: Revista de Medios y Educación, 46, pp. 103-117. doi: $12795 /$ pixelbit.2015.i46.07

More, C. (2008). Digital Stories Targeting Social Skills for Children with Disabilities: Multidimensional Learning. Intervention in School and Clinic, 43(3), pp. 168-177. doi: $\underline{10.1177 / 1053451207312919}$

Murillo García, J. L. (2010). Programas Escuela 2.0 y Pizarra Digital: un paradigma de mercantilización del sistema educativo a través de las TICs. REIFOP. Revista Electrónica Interuniversitaria de Formación del Profesorado, 13(2), pp. 65-78. Recuperado de http://www.aufop.com/aufop/uploaded_files/articulos/1278785070.pdf.

Núñez, P., García, M., \& Hermida, L. (2012). Tendencias de las relaciones sociales e interpersonales de los nativos digitales y jóvenes en la web 2.0. Revista latina de comunicación social, 67, pp. 1-15.

OCDE (2009). TALIS. Estudio Internacional sobre la Enseñanza y el Aprendizaje. Madrid: Ministerio de Educación (Informe español 2009). Recuperado de http://www.mecd.gob.es/dctm/cee/encuentros/xxiencuentro/xxieccee04talis2009.pdf?documentId=0901e72b813f69f1. doi: https://doi.org/10.1787/9789264068780-en.

OCDE (2013). PIS A 2012. Programa para la Evaluación Internacional de los Alumnos (Informe español). Vol. II. Madrid: Ministerio de Educación, Cultura y Deporte. Recuperado de http://www.mecd.gob.es/dctm/inee/internacional/pisa2012/pisa2012lineavolumenii.pdf?documentId=0901e72b817ab56d. 
OCDE (2014). Talis 2013. Estudio internacional de la enseñanza y el aprendizaje (Informe español). Madrid: Ministerio de Educación, Cultura y Deporte. Recuperado de http://www.mecd.gob.es/dctm/inee/internacional/talis2013/talispublicacionessep2014.pdf?documentId=0901e72b81adaba0. doi: https://doi.org/10.1787/9789264214293-fr.

Orden ECD/65/2015, de 21 de enero, por la que se describen las relaciones entre las competencias, los contenidos y los criterios de evaluación de la educación primaria, la educación secundaria obligatoria y el bachillerato. Boletín Oficial del Estado, 25, de 29 de enero de 2015. Recuperado de https://www.boe.es/boe/dias/2015/01/29/pdfs/BOE-A-2015-738.pdf.

Orit, E. H., \& Shrieber, B. (2004). Word Processing as an Assistive Technology Tool for Enhancing Academic Outcomes of Students with Writing Disabilities in the General Classroom. Journal of Learning Disabilities, 37(2), pp. 143-154. doi: 10.1177/00222194040370020501

Padilha, M. (2009). Tipos de indicadores: una mirada reflexiva. En R. Carneiro, J. C. Toscano y T. Díaz (coords.), Los desafios de las TIC para el cambio educativo (pp. 45-58). Madrid: Fundación Santillana.

Pérez-Escoda, A., Castro-Zubizarreta, A., \& Fandos, M. (2016). La competencia digital de la Generación Z: claves para su introducción curricular en la Educación Primaria. Comunicar, 49, pp. 7179. doi: $10.3916 / C 49-2016-07$

Prensky, M. (2001). Digital Natives, Digital Immigrants. On the Horizon, 9(5). Recuperado de http://www.marcprensky.com/writing/Prensky $\% 20 \% 20$ Digital $\% 20$ Natives, $\% 20$ Digital $\% 20$ Immigrants $\% 20-\% 20$ Part1.pdf.

Prensky, M. (2015). El mundo necesita un nuevo curriculo: habilidades para pensar, crear, relacionarse y actuar. Madrid: Ediciones SM.

Quicios, M. P. (2015). Los dispositivos digitales móviles en educación superior: usos y experiencias En E. Vázquez-Cano y M. L. Sevillano (coords.), Dispositivos digitales móviles en educación. El aprendizaje ubicuo (pp. 49-66). Madrid: Narcea.

Ramírez, A., \& González, N. (2016). Competencia mediática del profesorado y del alumnado de educación obligatoria en España. Comunicar, 49, pp. 49-58. doi: 10.3916/C49-2016-05

Real Decreto 126/2014, de 28 de febrero, por el que se establece el currículo básico de la Educación Primaria. Boletín Oficial del Estado, 52, de 1 de marzo de 2014. Recuperado de https://www.boe.es/boe/dias/2014/03/01/pdfs/BOE-A-2014-2222.pdf.

Recomendación 2006/962/CE del Parlamento Europeo y del Consejo, de 18 de diciembre, sobre las competencias clave para el aprendizaje permanente. Diario Oficial de la Unión Europea, L 394/10, de 30 de diciembre de 2006. Recuperado de https://www.boe.es/doue/2006/394/L0001000018.pdf.

Reig Hernández, D. (2003). El futuro de la educación superior, algunas claves. REIRE. Revista d'Innovació y Recerca en Educació, 3(2), pp. 98-115.

Ressa, T. W. (2010). The effects of a Word Prediction Program on the Number of Words Written by a Learner with Disabilities. Tesis doctoral. The Ohio State University.

Rioseco, M. H., \& Roig, R. (2014). Las expectativas hacia la integración de las TIC en educación desde una perspectiva fenomenológica. IJERI. International Journal of Educational Research and Innovation, 1, pp. 29-40. Recuperado de https://www.upo.es/revistas/index.php/IJERI/article/view/854/837.

Sáez Vacas, F. (2011). Nativos digitales, inteligencia digital. ¿Homo digitalis? Revista TELOS (Cuadernos de Comunicación e Innovación, 86, pp. 6-8. Recuperado de https://telos.fundaciontelefonica.com/url-direct/pdf-generator?tipoContenido=articuloTelos\&idContenido $=2011012711540001$ \&idioma $=$ es.

Santos, M. (2010). Pedagogía, historia y alteridad. Teoría de la Educación. Revista Interuniversitaria, 22(2), pp. 63-84.

Siemens, G. (2004). Connectivism: A Learning Theory for the Digital Age. Recuperado de http://elearnspace.org/Articles/connectivism.htm.

Siemens, G. (2010). Conociendo el conocimiento. Editorial digital Nodos Ele.

Small, G. (2009). El cerebro digital. Cómo las nuevas tecnologias están cambiando nuestra mente. Barcelona: Urano.

UNESCO (2002). Information and Communication Technology in Education. A curriculum for Schools and Programme of Teacher Development. París: UNESCO. Recuperado de http://unesdoc.unesco.org/images/0012/001295/129538e.pdf. 
UNESCO (2014). Enfoques estratégicos sobre las TIC en educación en América Latina y el Caribe. Santiago de Chile: UNESCO. Recuperado de http://unesdoc.unesco.org/images/0022/002232/223251s.pdf.

Urueña, S. (2016). Dimensiones de la inclusión de las TIC en el currículo educativo: una aproximación teórica. Teoría de la Educación. Revista interuniversitaria, 28(1), pp. 209-223. doi: 10.14201/teoredu2016281209223

Vallor, S. (2016). Technology and the Virtues: a philosophical guide to a future worth wanting. Oxford: Oxford University Press. doi: 10.1093/acprof:oso/9780190498511.001.0001

Valverde Berrocoso, J., Fernández Sánchez, M. R., y Revuelta Domínguez, F. I. (2013). El bienestar subjetivo ante las buenas prácticas educativas con TIC: su influencia en profesorado innovador. Educación XXI, 16(1), pp. 255-279. doi: 10.5944/educxx1.16.1.726

Vivancos, J. (2008). Tratamiento de la información y competencia digital. Madrid: Alianza. 